\title{
Optimization conditions for some fractional problems
}

\author{
Ricardo Almeida \\ CIDMA, Department of Mathematics, University of Aveiro \\ ricardo.almeida@ua.pt
}

\begin{abstract}
The purpose of this study is to present necessary conditions for calculus of variations problems, where the Lagrange function involves a Caputo fractional derivative with nonconstant order. The fractional operator depends on another function, and for particular choices of that function, we obtain some well-known fractional derivatives. Several necessary conditions of optimality are proven, namely the Euler-Lagrange equation.
\end{abstract}

Index Terms-Calculus of variations, fractional calculus, variable order derivative, Euler-Lagrange equation.

\section{INTRODUCTION}

Non-integer order derivatives are an old branch of mathematics, and go back to the birth of differential calculus [29], [35]. In 1695, Leibniz asked L'Hôpital: "Can the meaning of derivatives with integer order be generalized to derivatives with non-integer orders?" Later, L'Hôpital answered him: "What if the order is $1 / 2$ ?". Since then, the field of fractional calculus has been studied extensively, though at the beginning mainly in pure mathematics. More recently, it has been applied in several areas such as science, economy, engineering, etc. To name a few, we mention some applications to control theory [30], [37], electrical circuits [26], heat conduction [18], diffusion equations [22], [23], sound wave propagation [15], etc. In this work, we consider variational problems, replacing integer order derivatives by fractional order derivatives in the problem. In [33], it was proven that fractional calculus can deal better with dissipative problems, where an Euler-Lagrange equation was obtained. Since then, numerous works have appeared dealing with different fractional integrals/derivatives. For example, for derivatives, some of the definitions are the Riemann-Liouville [1], [11], [16], the Caputo [2], [9], [24], the symmetric [20], the Riesz fractional derivative [3], [10], the generalized fractional derivative [27], [28], and with respect to another function [6]. We also mention the books [8], [25], where numerical and analytical methods are presented to solve fractional variational problems.

Let $\Upsilon \in L^{1}([a, b], \mathbb{R})$. The most important operators are:

1) the Gamma function $\Gamma$.

2) Riemann-Liouville integral $(\nu>0)$ :

$$
I_{a+}^{\nu} \Upsilon(t):=\frac{1}{\Gamma(\nu)} \int_{a}^{t}(t-\chi)^{\nu-1} \Upsilon(\chi) d \chi .
$$

This work was supported by Portuguese funds through the CIDMA, and FCT-Fundação para a Ciência e a Tecnologia- UID/MAT/04106/2013.

978-1-5386-5346-6/18/\$31.00 () 2018 IEEE
3) Riemann-Liouville derivative $(\nu>0)$ :

$$
D_{a+}^{\nu} \Upsilon(t):=\left(\frac{d}{d t}\right)^{n} I_{a+}^{n-\nu} \Upsilon(t),
$$

where $n$ is the ceiling of $\nu$.

4) Caputo derivative [13] $(\nu>0)$ :

$$
{ }^{C} D_{a+}^{\nu} \Upsilon(t):=I_{a+}^{n-\nu} \Upsilon^{(n)}(t),
$$

where $n$ is the ceiling of $\nu$ if $\nu \notin \mathbb{N}$ and $n=\nu$ otherwise.

However, these are not the only existent definitions, and many others can be found in the literature. It is worth to mention that, when $\nu$ is an integer number, let us say $\nu=m \in \mathbb{N}$, the fractional derivative operator is simply the usual derivative: ${ }^{C} D_{a+}^{m} \Upsilon(t)=D_{a+}^{m} \Upsilon(t)=\Upsilon^{(m)}(t)$

One way to reduce such number of concepts is to define fractional derivatives/integrals with respect to another function [35]. For more on the subject, we advice the reader to see [5]. Let $\nu>0$ be a real, $\Upsilon, k:[a, b] \rightarrow \mathbb{R}$ be two functions with $k$ differentiable and $k^{\prime}(t)>0$, for all $t$. The operators we are going to study are defined using another function. They are the following:

1) the $k$-fractional integral:

$$
I_{a+}^{\nu, k} \Upsilon(t):=\frac{1}{\Gamma(\nu)} \int_{a}^{t} k^{\prime}(\chi)(k(t)-k(\chi))^{\nu-1} \Upsilon(\chi) d \chi
$$

2) the $k$-Riemann-Liouville fractional derivative:

$$
D_{a+}^{\nu, k} \Upsilon(t):=\left(\frac{1}{k^{\prime}(t)} \frac{d}{d t}\right)^{n} I_{a+}^{n-\nu, k} \Upsilon(t),
$$

where $n=[\nu]+1$.

3) the $k$-Caputo fractional derivative:

$$
{ }^{C} D_{a+}^{\nu, k} \Upsilon(t):=I_{a+}^{n-\nu, k}\left(\frac{1}{k^{\prime}(t)} \frac{d}{d t}\right)^{n} \Upsilon(t),
$$

where $n=\lceil\nu\rceil$ if $\nu \notin \mathbb{N}$, and $n=\nu$ otherwise.

We mention that, if $\nu=m$ is an integer number, then ${ }^{C} D_{a+}^{m, k} \Upsilon(t)=\left(\frac{1}{k^{\prime}(t)} \frac{d}{d t}\right)^{m} \Upsilon(t)$. If $\nu$ is not an integer, then

$$
\begin{aligned}
&{ }^{C} D_{a+}^{\nu, k} \Upsilon(t)=\frac{1}{\Gamma(n-\nu)} \int_{a}^{t}(k(t)-k(\chi))^{n-\nu-1} k^{\prime}(\chi) \\
& \times\left(\frac{1}{k^{\prime}(\chi)} \frac{d}{d \chi}\right)^{n} \Upsilon(\chi) d \chi
\end{aligned}
$$


For special choices of $k$, we obtain the Caputo (when we consider $k(t)=t$ ), the Caputo-Hadamard (when the kernel is the function $k(t)=\ln (t))$, the Caputo-Katugampola $\left(k(t)=t^{\rho}\right)$ and the Caputo-Erdelyi-Kober (similar to the previous case) fractional derivatives. Recently, the purposed general form of differentiation has found applications (see [4], [7], [17], [39]).

One extension of these works is to consider the order of the derivative not constant along the process [36]. Since most problems are dynamic processes, it makes sense that the order of the operator varies with the process. Recently, it has found applications in control, physics, etc [14], [19], [32], [38].

The objective is to formulate conditions for variational problems, where the derivative operator is defined with respect to another function and the order of the derivative is nonconstant. In this way, we present some generalizations of known theorems in the calculus of variations, for the fractional context. For special cases of $k$ and $\nu$, we obtain classic results of this theory. We present and prove several necessary conditions that allow us to find the possible extremizers of the functionals. This study started with Riewe [33], [34]. As Riewe refered, "Traditional Lagrangian and Hamiltonian mechanics cannot be used with nonconservative forces such as friction", and after it numerous works have come up dealing with different problems. Applications are being found nowadays, in particular in fractional optimal control problems [12], [21], [31], [40]

The organization is the following. In Section II we present the needed definitions and results. Next, in Section III, we present some results on necessary conditions for minimization of a functional, and we end with a Conclusion section.

\section{SOME DEFINITIONS ON FRACTIONAL CALCULUS}

Throughout this paper, $\nu$ is a function with domain $[a, b]$ taking values between zero and one ( $\nu$ is the fractional order). Also, $x$ and $k$ are two differentiable functions, defined on $[a, b]$, and $k$ is increasing with $k^{\prime}>0$. The $k$-Caputo fractional derivative (of order $\nu(\cdot)$ ), is given by

${ }^{C} D_{a+}^{\nu(t), k} \Upsilon(t)=\frac{1}{\Gamma(1-\nu(t))} \int_{a}^{t}(k(t)-k(\chi))^{-\nu(t)} \Upsilon^{\prime}(\chi) d \chi$.

For the power function $\Upsilon(t)=(k(t)-k(a))^{\xi}(\xi>0)$ :

$$
{ }^{C} D_{a+}^{\nu(t), k} \Upsilon(t)=\frac{\Gamma(\xi+1)}{\Gamma(\xi-\nu(t)+1)}(k(t)-k(a))^{\xi-\nu(t)} .
$$

For example, let $\Upsilon(t)=(k(t)-k(0))^{2}$, with $t \in[0,2]$. For fractional orders, we consider $\nu_{1}(t)=0.5, \nu_{2}(t)=(t+1) / 4$, and $\nu_{3}(t)=\exp (t) / 8$. In Figures (1)-(2) we present the plots of ${ }^{C} D_{0+}^{\nu_{i}(t), k} \Upsilon(t)$ when considering $k(t)=t^{3 / 2}$ and $k(t)=$ $\ln (t+1)$, respectively.

Next, we present and prove an integration by parts formula, needed for the sequel. First, let us present two more definitions:

$$
I_{b-}^{\nu(t), k} \Upsilon(t):=\int_{t}^{b} \frac{k^{\prime}(\chi)}{\Gamma(\nu(\chi))}(k(\chi)-k(t))^{\nu(\chi)-1} \Upsilon(\chi) d \chi,
$$

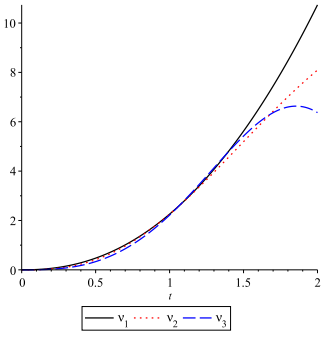

Fig. 1. Plots of ${ }^{C} D_{0+}^{\nu_{i}(t), k} \Upsilon(t)$ with $k(t)=t^{3 / 2}$.

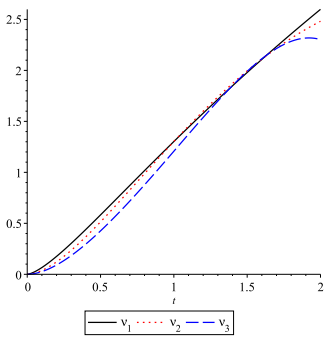

Fig. 2. Plots of ${ }^{C} D_{0+}^{\nu_{i}(t), k} \Upsilon(t)$ with $k(t)=\ln (t+1)$.

and

$$
\begin{aligned}
D_{b-}^{\nu(t), k} \Upsilon(t):=\frac{1}{k^{\prime}(t)} \frac{d}{d t} \int_{t}^{b} & \frac{-k^{\prime}(\chi)}{\Gamma(1-\nu(\chi))} \\
& \times(k(\chi)-k(t))^{-\nu(\chi)} \Upsilon(\chi) d \chi
\end{aligned}
$$

Theorem 1. Let $\Upsilon \in C[a, b]$ and $\Phi \in C^{1}[a, b]$. Then,

$$
\begin{array}{r}
\int_{a}^{b} \Upsilon(t)^{C} D_{a+}^{\nu(t), k} \Phi(t) d t=\left[I_{b-}^{1-\nu(t), k}\left(\frac{\Upsilon(t)}{k^{\prime}(t)}\right) \Phi(t)\right]_{a}^{b} \\
+\int_{a}^{b} D_{b-}^{\nu(t), k}\left(\frac{\Upsilon(t)}{k^{\prime}(t)}\right) \Phi(t) k^{\prime}(t) d t
\end{array}
$$

Proof. First, observe that

$$
\begin{aligned}
& \int_{a}^{b} \Upsilon(t)^{C} D_{a+}^{\nu(t), k} \Phi(t) d t=\int_{a}^{b} \int_{a}^{t} \frac{\Upsilon(t)}{\Gamma(1-\nu(t))} \\
& \quad \times(k(t)-k(\chi))^{-\nu(t)} \Phi^{\prime}(\chi) d \chi d t \\
& =\int_{a}^{b} \int_{t}^{b} \frac{\Upsilon(\chi)}{\Gamma(1-\nu(\chi))}(k(\chi)-k(t))^{-\nu(\chi)} d \chi \cdot \Phi^{\prime}(t) d t .
\end{aligned}
$$


Consequently, we obtain

$$
\begin{gathered}
{\left[\left(\int_{t}^{b} \frac{\Upsilon(\chi)}{\Gamma(1-\nu(\chi))}(k(\chi)-k(t))^{-\nu(\chi)} d \chi\right) \Phi(t)\right]_{a}^{b}} \\
-\int_{a}^{b} \frac{d}{d t}\left(\int_{t}^{b} \frac{\Upsilon(\chi)}{\Gamma(1-\nu(\chi))}(k(\chi)-k(t))^{-\nu(\chi)} d \chi\right) \Phi(t) d t
\end{gathered}
$$

proving the desired formula.

\section{CAlCulus of VARiations OF FRACTIONAL ORDER}

In this section, we intend to present some conditions to determine the optimal solutions. The Lagrangian depends of the Caputo derivative, and the order of the derivative is not constant. The problem is the following:

$$
\begin{aligned}
\mathcal{F}: C^{1}[a, b] & \rightarrow \mathbb{R} \\
x & \mapsto \int_{a}^{b} Z\left(t, x(t),{ }^{C} D_{a+}^{\nu(t), k} x(t)\right) d t .
\end{aligned}
$$

The function $Z:[a, b] \times \mathbb{R}^{2} \rightarrow \mathbb{R}, Z=Z(t, x, v)$ is continuously differentiable for $x$ and $v, \partial_{2} Z$ and $\partial_{3} Z$. The boundary conditions

$$
x(a)=\theta, \quad x(b)=\vartheta, \quad \theta, \vartheta \in \mathbb{R}
$$

may be imposed to the problem. The next theorem presents a necessary condition to determine the candidates for minimizers of the functional. Such type of equations are usually called Euler-Lagrange equations. Along our work we will assume that the map $t \mapsto D_{b-}^{\nu(t), k}\left(\partial_{3} Z\left(t, x(t),{ }^{C} D_{a+}^{\nu(t), k} x(t)\right) / k^{\prime}(t)\right)$ is continuous in $[a, b]$, where $x$ is a minimizer of the functional. Also, we will use the following notations. By $\{x\}(t)$, we mean the 3 -dimensional vector $\left(t, x(t),{ }^{C} D_{a+}^{\nu(t), k} x(t)\right)$. By $\partial_{i} f\left(z_{1}, \ldots, z_{m}\right)$, we mean

$$
\partial_{i} f\left(z_{1}, \ldots, z_{m}\right):=\frac{\partial f}{\partial z_{i}}\left(z_{1}, \ldots, z_{m}\right) .
$$

Theorem 2. Suppose that $x$ is a curve that minimizes $\mathcal{F}(1)$, defined on a set of functions that fulfill the condition (2). Then, $x$ satisfies the following equation

$$
\partial_{2} Z\{x\}(t)+D_{b-}^{\nu(t), k}\left(\frac{\partial_{3} Z\{x\}(t)}{k^{\prime}(t)}\right) k^{\prime}(t)=0,
$$

for all $t \in[a, b]$.

Proof. To prove this result, we start by considering a new function $\varpi \in C^{1}[a, b]$. Since $x$ is fixed at the endpoints $a$ and $b$, we must impose the conditions $\varpi(a)=0$ and also $\varpi(b)=0$. Since

$$
\int_{a}^{b}\left[\partial_{2} Z\{x\}(t) \varpi(t)+\partial_{3} Z\{x\}(t)^{C} D_{a+}^{\nu(t), k} \varpi(t)\right] d t=0,
$$

applying Theorem 1, we can deduce that

$$
\begin{gathered}
\int_{a}^{b}\left[\partial_{2} Z\{x\}(t)+D_{b-}^{\nu(t), k}\left(\frac{\partial_{3} Z\{x\}(t)}{k^{\prime}(t)}\right) k^{\prime}(t)\right] \varpi(t) d t \\
+\left[I_{b-}^{1-\nu(t), k}\left(\frac{\partial_{3} Z\{x\}(t)}{k^{\prime}(t)}\right) \varpi(t)\right]_{a}^{b}=0 .
\end{gathered}
$$

Since $\varpi$ is arbitrary in the open interval $] a, b[$, we prove formula (3).

Theorem 3. If $\mathcal{F}$ attains a minimum value at $x$, then $x$ satisfies Eq. (3) and the two transversality conditions

$$
I_{b-}^{1-\nu(t), k}\left(\frac{\partial_{3} Z\{x\}(t)}{k^{\prime}(t)}\right)=0
$$

evaluated at the endpoints of the interval $[a, b]$.

Proof. Observe that $\varpi$ is arbitrary in the closed interval $[a, b]$, obtaining the desired formulas.

The case of functionals depending on a vector function $x:=$ $\left(x_{1}, \ldots, x_{m}\right)$ is similar to the previous ones. Now, the fractional derivative of the vector $x$ is defined as ${ }^{C} D_{a+}^{\nu(t), k} x(t):=$ $\left({ }^{C} D_{a+}^{\nu(t), k} x_{1}(t), \ldots,{ }^{C} D_{a+}^{\nu(t), k} x_{m}(t)\right)$.

Theorem 4. Let $x=\left(x_{1}, \ldots, x_{m}\right)$ be a minimizer of

$$
\mathcal{F}\left(x_{1}, \ldots, x_{m}\right):=\int_{a}^{b} Z\{x\}(t) d t .
$$

Then, for every $t$,

$$
\partial_{i+1} Z\{x\}(t)+D_{b-}^{\nu(t), k}\left(\frac{\partial_{i+1+m} Z\{x\}(t)}{k^{\prime}(t)}\right) k^{\prime}(t)=0,
$$

where the index $i=1, \ldots, m$. Moreover, if $x_{i}(a)$ is free, then

$$
I_{b-}^{1-\nu(t), k}\left(\frac{\partial_{i+1+m} Z\{x\}(t)}{k^{\prime}(t)}\right)=0
$$

at $t=a$, and if $x_{i}(b)$ is free, then

$$
I_{b-}^{1-\nu(t), k}\left(\frac{\partial_{i+1+m} Z\{x\}(t)}{k^{\prime}(t)}\right)=0
$$

at $t=b$.

So far, both the fractional derivative and the cost integral (1) start at the same point $t=a$. The next theorem generalizes Theorem 2 , by considering the case when the integral starts at a point $t=A>a$.

Theorem 5. Let

$$
\mathcal{F}(x):=\int_{A}^{b} Z\{x\}(t) d t,
$$

where $A>a$ is a fixed real. If $x$ minimizes $\mathcal{F}$, then,

$$
D_{b-}^{\nu(t), k}\left(\frac{\partial_{3} Z\{x\}(t)}{k^{\prime}(t)}\right)-D_{A-}^{\nu(t), k}\left(\frac{\partial_{3} Z\{x\}(t)}{k^{\prime}(t)}\right)=0,
$$


2018 13th APCA International Conference on Automatic Control and Soft Computing (CONTROLO)

June 4-6, 2018, Ponta Delgada, Azores, Portugal

for all $t \in[a, A]$, and for all $t \in[A, b]$, we also have the following:

$$
\partial_{2} Z\{x\}(t)+D_{b-}^{\nu(t), k}\left(\frac{\partial_{3} Z\{x\}(t)}{k^{\prime}(t)}\right) k^{\prime}(t)=0 .
$$

Moreover, we have following transversality conditions hold:

$$
\begin{gathered}
I_{b-}^{1-\nu(t), k}\left(\frac{\partial_{3} Z\{x\}(t)}{k^{\prime}(t)}\right)=0, \quad \text { at } t=b, \\
I_{b-}^{1-\nu(t), k}\left(\frac{\partial_{3} Z\{x\}(t)}{k^{\prime}(t)}\right) \\
-I_{A-}^{1-\nu(t), k}\left(\frac{\partial_{3} Z\{x\}(t)}{k^{\prime}(t)}\right)=0, \quad \text { at } t=a,
\end{gathered}
$$

and

$$
I_{A-}^{1-\nu(t), k}\left(\frac{\partial_{3} Z\{x\}(t)}{k^{\prime}(t)}\right)=0, \quad \text { at } t=A .
$$

Proof. Using the following relations

$$
\begin{aligned}
& 0=\int_{A}^{b}\left[\partial_{2} Z\{x\}(t) \varpi(t)+\partial_{3} Z\{x\}(t)^{C} D_{a+}^{\nu(t), k} \varpi(t)\right] d t \\
& =\int_{a}^{b}\left[\partial_{2} Z\{x\}(t) \varpi(t)+\partial_{3} Z\{x\}(t)^{C} D_{a+}^{\nu(t), k} \varpi(t)\right] d t \\
& -\int_{a}^{A}\left[\partial_{2} Z\{x\}(t) \varpi(t)+\partial_{3} Z\{x\}(t)^{C} D_{a+}^{\nu(t), k} \varpi(t)\right] d t \\
& =\int_{a}^{b}\left[\partial_{2} Z\{x\}(t)+D_{b-}^{\nu(t), k}\left(\frac{\partial_{3} Z\{x\}(t)}{k^{\prime}(t)}\right) k^{\prime}(t)\right] \varpi(t) d t \\
& -\int_{a}^{A}\left[\partial_{2} Z\{x\}(t)+D_{A-}^{\nu(t), k}\left(\frac{\partial_{3} Z\{x\}(t)}{k^{\prime}(t)}\right) k^{\prime}(t)\right] \varpi(t) d t \\
& +\left[I_{b-}^{1-\nu(t), k}\left(\frac{\partial_{3} Z\{x\}(t)}{k^{\prime}(t)}\right) \varpi(t)\right]_{a}^{b} \\
& -\left[I_{A-}^{1-\nu(t), k}\left(\frac{\partial_{3} Z\{x\}(t)}{k^{\prime}(t)}\right) \varpi(t)\right]_{a}^{A} \\
& =\int_{a}^{A}\left[D_{b-}^{\nu(t), k}\left(\frac{\partial_{3} Z\{x\}(t)}{k^{\prime}(t)}\right)-D_{A-}^{\nu(t), k}\left(\frac{\partial_{3} Z\{x\}(t)}{k^{\prime}(t)}\right)\right] \\
& \times k^{\prime}(t) \varpi(t) d t \\
& +\int_{A}^{b}\left[\partial_{2} Z\{x\}(t)+D_{b-}^{\nu(t), k}\left(\frac{\partial_{3} Z\{x\}(t)}{k^{\prime}(t)}\right) k^{\prime}(t)\right] \varpi(t) d t \\
& +\left[I_{b-}^{1-\nu(t), k}\left(\frac{\partial_{3} Z\{x\}(t)}{k^{\prime}(t)}\right) \varpi(t)\right]_{a}^{b} \\
& -\left[I_{A-}^{1-\nu(t), k}\left(\frac{\partial_{3} Z\{x\}(t)}{k^{\prime}(t)}\right) \varpi(t)\right]_{a}^{A},
\end{aligned}
$$

and for appropriate choices of $\varpi$, we obtain the desired result.

For our next result, besides the boundary conditions (2), we impose an integral constraint of type

$$
\mathcal{P}(x):=\int_{a}^{b} P\left(t, x(t),{ }^{C} D_{a+}^{\nu(t), k} x(t)\right) d t=K,
$$

for a fixed real $K$. Again, we assume that $P$ is differentiable with respect to $x$ and $v$, and $t \mapsto D_{b-}^{\nu(t), k}\left(\partial_{3} P\{x\}(t) / k^{\prime}(t)\right)$ is continuous in $[a, b]$.

Theorem 6. Suppose that $\mathcal{F}$ (1), subject to the restrictions (2) and (4), attains a minimum value at $x$. If $x$ is not a solution of

$$
\partial_{2} P\{x\}(t)+D_{b-}^{\nu(t), k}\left(\frac{\partial_{3} P\{x\}(t)}{k^{\prime}(t)}\right) k^{\prime}(t)=0, \quad \forall t \in[a, b],
$$

then there is $\lambda \in \mathbb{R}$ for which $x$ is a solution of

$$
\partial_{2} F\{x\}(t)+D_{b-}^{\nu(t), k}\left(\frac{\partial_{3} F\{x\}(t)}{k^{\prime}(t)}\right) k^{\prime}(t)=0, \quad \forall t \in[a, b],
$$

where $F:=Z+\lambda P$.

Proof. Define the two following auxiliary functions

$$
j\left(\epsilon_{1}, \epsilon_{2}\right):=\mathcal{F}\left(x+\epsilon_{1} \varpi_{1}+\epsilon_{2} \varpi_{2}\right)
$$

and

$$
p\left(\epsilon_{1}, \epsilon_{2}\right):=\mathcal{P}\left(x+\epsilon_{1} \varpi_{1}+\epsilon_{2} \varpi_{2}\right)-K,
$$

where $\varpi_{i} \in C^{1}[a, b]$ and $\varpi_{i}(a)=0=\varpi_{i}(b)$, for $i=1,2$. Since

$$
\begin{aligned}
& \partial_{1} p(0,0)= \\
& \int_{a}^{b}\left[\partial_{2} P\{x\}(t)+D_{b-}^{\nu(t), k}\left(\frac{\partial_{3} P\{x\}(t)}{k^{\prime}(t)}\right) k^{\prime}(t)\right] \varpi_{1}(t) d t
\end{aligned}
$$

and $x$ does not satisfies Eq. (5), there is a function $\varpi_{1} \in$ $C^{1}[a, b]$ with $\partial_{1} p(0,0) \neq 0$. On the other hand, $(0,0)$ is a minimizer of $j$, with the restriction $p(\cdot, \cdot)=0$, Thus, we ensure the existence of $\lambda \in \mathbb{R}$ with

$$
\nabla(j+\lambda p)(0,0)=(0,0) .
$$

Computing $\partial_{2}(j+\lambda p)(0,0)$, we prove the theorem.

Next, we consider an holonomic constraint of type

$$
g(t, x(t))=0, \quad \forall t \in[a, b] .
$$

The function $x$ is a two dimensional vector, that is, we are dealing with a function $x$ of form $x(t)=\left(x_{1}(t), x_{2}(t)\right)$. The function $g:[a, b] \times \mathbb{R}^{2} \rightarrow \mathbb{R}$ is differentiable with respect to $x_{1}$ and $x_{2}$. We also consider the conditions

$$
x(a)=\theta \quad \text { and } \quad x(b)=\vartheta, \quad \theta, \vartheta \in \mathbb{R}^{2} .
$$

Theorem 7. Let

$$
\mathcal{F}(x)=\int_{a}^{b} Z\left(t, x(t),{ }^{C} D_{a+}^{\nu(t), k} x(t)\right) d t,
$$

subject to the constraints (6)-(7). Suppose that $x$ is a minimizer for $\mathcal{F}$ and that, for every $t \in[a, b]$,

$$
\partial_{3} g(t, x(t)) \neq 0 \text {. }
$$

The existence of a continuous function $\Xi:[a, b] \rightarrow \mathbb{R}$ is ensured for which $x$ verifies the equation

$$
\partial_{i+1} Z\{x\}(t)+D_{b-}^{\nu(t), k}\left(\frac{\partial_{i+3} Z\{x\}(t)}{k^{\prime}(t)}\right) k^{\prime}(t)
$$




$$
+\Xi(t) \partial_{i+1} g(t, x(t))=0,
$$

for all $t$ and $i=1,2$.

Proof. Let $\varpi$ be a differentiable function with domain $[a, b]$, $\varpi=\left(\varpi_{1}, \varpi_{2}\right)$. By the imposed restrictions on the bounds of the domain, the conditions $\varpi(a)=(0,0)$ and $\varpi(b)=(0,0)$ are assumed. From Eq. (8),

$$
g\left(t, x_{1}(t)+\epsilon \varpi_{1}(t), x_{2}(t)+\epsilon \varpi_{2}(t)\right)=0, \quad \forall t \in[a, b] .
$$

If we differentiate Eq. (10) to the variable $\epsilon$, and after replacing $\epsilon=0$, we get

$$
\partial_{3} g(t, x(t)) \varpi_{2}(t)=-\partial_{2} g(t, x(t)) \varpi_{1}(t) .
$$

Let us define $\Xi:[a, b] \rightarrow \mathbb{R}$ as

$$
\Xi(t):=-\frac{\partial_{3} Z\{x\}(t)+D_{b-}^{\nu(t), k}\left(\frac{\partial_{5} Z\{x\}(t)}{k^{\prime}(t)}\right) k^{\prime}(t)}{\partial_{3} g(t, x(t))} .
$$

Observe that Eq. (9) is proven for $i=2$. For the case $i=1$, using Eqs. (11)-(12), we get that

$$
\begin{gathered}
\left(\partial_{3} Z\{x\}(t)+D_{b-}^{\nu(t), k}\left(\frac{\partial_{5} Z\{x\}(t)}{k^{\prime}(t)}\right) k^{\prime}(t)\right) \varpi_{2}(t) \\
=\Xi(t) \partial_{2} g(t, x(t)) \varpi_{1}(t) .
\end{gathered}
$$

Since the first derivative of $\mathcal{J}$ must vanish at $x$, using integration by parts and Eq. (13), we prove the remaining case.

\section{EXAMPLES}

Let

$$
\begin{aligned}
\mathcal{F}(x)= & \int_{a}^{b}\left({ }^{C} D_{a+}^{\nu(t), k} x(t)\right. \\
& \left.-\frac{8 !}{\Gamma(9-\nu(t))}(k(t)-k(a))^{8-\nu(t)}\right)^{2} d t \rightarrow \min
\end{aligned}
$$

with the restrictions

$$
x(a)=0, \quad x(b)=(k(b)-k(a))^{8} .
$$

Since $\mathcal{J}(x) \geq 0$, for an arbitrary $x$, and $\mathcal{J}(\iota)=0$ for $\iota:=$ $(k(t)-k(a))^{8}$, we conclude easily that the solution is the power function $\iota$. Let us see that $\iota$ satisfies the Euler-Lagrange equation (3). In fact, Eq. (3) becomes

$$
D_{b-}^{\nu(t), k} \frac{{ }^{C} D_{a+}^{\nu(t), k} x(t)-\frac{8 !}{\Gamma(9-\nu(t))}(k(t)-k(a))^{8-\nu(t)}}{k^{\prime}(t)}=0 .
$$

Since

$$
{ }^{C} D_{a+}^{\nu(t), k} \iota(t)=\frac{8 !}{\Gamma(9-\nu(t))}(k(t)-k(a))^{8-\nu(t)},
$$

it is an easy exercise to verify that $\iota$ is a candidate for solution.

As second example, we will deal with an isoperimetric question:

$$
\begin{aligned}
\mathcal{F}(x)= & \int_{a}^{b}\left({ }^{C} D_{a+}^{\nu(t), k} x(t)\right)^{2} \\
& +\left(\frac{8 !}{\Gamma(9-\nu(t))}(k(t)-k(a))^{8-\nu(t)}\right)^{2} d t \rightarrow \min
\end{aligned}
$$

subject to

$$
x(a)=0, \quad x(b)=(k(b)-k(a))^{8},
$$

and to the integral constraint

$$
\int_{a}^{b}{ }^{C} D_{a+}^{\nu(t), k} x(t) \frac{8 !}{\Gamma(9-\nu(t))}(k(t)-k(a))^{8-\nu(t)} d t=K
$$

where

$$
K=\int_{a}^{b}\left(\frac{8 !}{\Gamma(9-\nu(t))}(k(t)-k(a))^{8-\nu(t)}\right)^{2} d t .
$$

Again, $\iota:=(k(t)-k(a))^{8}$ verifies the required conditions given by Theorem 6 with $\lambda=-2$.

\section{CONCLUSION}

We studied some calculus of variations problems. Instead of considering Lagrangians depending on the first order derivative, we replace it by a fractional derivative with respect to $k$. Also, the order of the derivative is variable in time and takes any value in the interval $(0,1)$. An Euler-Lagrange equation was obtained, as well some other cases, namely the ones when in presence of an integral and holonomic constraints. The author wants to thank the reviewers for the positive remarks that helped to improve this work.

\section{REFERENCES}

[1] O. P. Agrawal, "Fractional variational calculus and the transversality conditions," J. Phys. A, vol. 39, pp. 10375-10384, 2006.

[2] O. P. Agrawal, "Generalized Euler-Lagrange equations and transversality conditions for FVPs in terms of the Caputo derivative," J. Vib. Control, vol. 13, pp. 1217-1237, 2007.

[3] O. P. Agrawal, "Fractional variational calculus in terms of Riesz fractional derivatives," J. Phys. A, vol. 40, pp. 6287-6303, 2007.

[4] R. Almeida, "What is the best fractional derivative to fit data?" Appl. Anal. Discrete Math., vol 11, pp. 358-368, 2017.

[5] R. Almeida, "A Caputo fractional derivative of a function with respect to another function," Commun. Nonlinear Sci. Numer. Simul., vol. 44, pp. $460-481,2017$.

[6] R. Almeida, "Optimality conditions for fractional variational problems with free terminal time," Discrete Contin. Dyn. Syst. Ser. S, vol. 11, no. 1, pp. 143-154, 2018.

[7] R. Almeida, A. B. Malinowska, and M. T. T. Monteiro, "Fractional differential equations with a Caputo derivative with respect to a kernel function and their applications," Math. Meth. Appl. Sci., vol. 41, pp. 336-352, 2018.

[8] R. Almeida, S. Pooseh, and D.F.M. Torres, Computational methods in the fractional calculus of variations, Imperial College Press, London 2015.

[9] D. Baleanu, "Fractional constrained systems and Caputo derivatives," J. Comput. Nonlinear Dyn., vol. 3, 021102, 2008.

[10] D. Baleanu, "New applications of fractional variational principles," Rep. Math. Phys., vol. 61, pp. 199-206, 2008.

[11] D. Baleanu, T. Maaraba, and F. Jarad, "Fractional variational principles with delay," J. Phys. A, vol. 41, 315403, 8 pp, 2008.

[12] D. Baleanu, R. Caponetto, and J.T. Machado, "Challenges in fractional dynamics and control theory," J. Vib. Control, vol. 22, pp. 2151-2152, 2016.

[13] M. Caputo, "Linear models of dissipation whose $Q$ is almost frequency independentII,” Geophys. JR Astr. Soc., vol. 13, pp. 529-539, 1967.

[14] C. F. M. Coimbra, C. M. Soon, and M. H. Kobayashi, "The variable viscoelasticity operator," Annalen der Physik, vol. 14, pp. 378-389, 2005.

[15] Z. E. A. Fellah and C. Depollier, "Application of fractional calculus to the sound waves propagation in rigid porous materials: Validation via ultrasonic measurement," Acta Acust., vol. 88, pp. 34-39, 2002. 
2018 13th APCA International Conference on Automatic Control and Soft Computing (CONTROLO)

June 4-6, 2018, Ponta Delgada, Azores, Portugal

[16] G. S. F. Frederico and D. F. M. Torres, "Fractional conservation laws in optimal control theory,” Nonlinear Dynam., vol. 53, pp. 215-222, 2008

[17] R. Garra, A. Giusti, and F. Mainardi, "The fractional Dodson diffusion equation: a new approach," (submitted).

[18] M. Goto and D. Ishii, "Semidifferential electroanalysis," J. Electroanal. Chem. and Interfacial Electrochem., vol 61, pp. 361-365, 1975.

[19] D. Ingman and J. Suzdalnitsky, "Control of damping oscillations by fractional differential operator with time-dependent order," Comput. Methods Appl. Mech. Engrg., vol. 193, no. 52, pp. 5585-5595, 2004.

[20] M. Klimek, "Fractional sequential mechanics-models with symmetric fractional derivatives," Czech. J. Phys., vol. 52, pp. 1247-1253 2002.

[21] M. Lazarević, "Some Applications of Biomimetics and Fractional Calculus in Control and Modeling of (Bio)robotic Systems," In: A. Rodić, D Pisla, and H. Bleuler (eds), New Trends in Medical and Service Robots, Mechanisms and Machine Science, vol 20. Springer, Cham, 2014.

[22] F. Mainardi, "The fundamental solutions for the fractional diffusionwave equation," Appl. Math. Lett., vol. 9, no. 6, pp. 23-28, 1996.

[23] F. Mainardi, "Fractional relaxation-oscillation and fractional diffusionwave phenomena," Chaos Solitons Fractals, vol. 7, pp. 1461-1477, 1996.

[24] A. B. Malinowska and D.F.M. Torres, "Generalized natural boundary conditions for fractional variational problems in terms of the Caputo derivative," Comput. Math. Appl., vol. 59, pp. 3110-3116, 2010.

[25] A. B. Malinowska and D.F.M. Torres, Introduction to the fractional calculus of variations, Imperial College Press, London, 2012.

[26] A. Le Mehaute and G. Crepy, "Introduction to transfer and motion in fractal media: the geometry of kinetics," Solid State Ion., vol. 9-10, pp. $17-30,1983$.

[27] T. Odzijewicz, A. B. Malinowska, and D. F. M. Torres, "A generalized fractional calculus of variations," Control Cybernet., vol. 42, pp. 443$458,2013$.

[28] T. Odzijewicz, A. B. Malinowska, and D. F. M. Torres, "Generalized fractional calculus with applications to the calculus of variations," Comput. Math. Appl., vol. 64, pp. 3351-3366, 2012.

[29] K. B Oldham and J. Spanier. The fractional calculus, Academic Press, New York, 1974.

[30] A. Oustaloup, Systèmes asservis linéaires d'ordre fractionnaire, Masson, Paris, 1983 (French)

[31] S.M.A. Pahnehkolaei, A. Alfi, and J.A.T. Machado, "Chaos suppression in fractional systems using adaptive fractional state feedback control," Chaos Solitons Fract., vol. 103, pp. 488-503, 2017.

[32] L. E. S. Ramirez and C. F. M. Coimbra, "On the variable order dynamics of the nonlinear wake caused by a sedimenting particle," Phys. D, vol. 240 , no. 13 , pp. 1111-1118, 2011.

[33] F. Riewe, "Nonconservative Lagrangian and Hamiltonian mechanics," Phys. Rev. E, vol. 53, pp. 1890-1899, 1996.

[34] F. Riewe, "Mechanics with fractional derivatives," Phys. Rev. E, vol. 55 pp. 3581-3592, 1997.

[35] S. G. Samko, A. A. Kilbas, and O. I. Marichev, Fractional integrals and derivatives, translated from the 1987 Russian original, Gordon and Breach, Yverdon, 1993.

[36] S. G. Samko and B. Ross, "Integration and differentiation to a variable fractional order," Integral Transform. Spec. Funct., vol. 1, no. 4, pp. 277-300, 1993

[37] J. I. Suárez, B. M. Vinagre , A. J. Calderón , C. A. Monje, and Y. Q. Chen, Using Fractional Calculus for Lateral and Longitudinal Contro of Autonomous Vehicles, in Lecture Notes in Computer Science, vol. 2809 Springer Berlin/Heidelberg, 2004.

[38] D. Valério and J. Sá da Costa, "Variable order fractional controllers," Asian J. Control, vol. 15, no. 3, pp. 648-657, 2013.

[39] X. J. Yang and J. A. Machado, "A new fractional operator of variable order: Application in the description of anomalous diffusion," Physica A, vol. 481, pp. 276283, 2017.

[40] M.A. Zaky, and J.A.T. Machado, "On the formulation and numerical simulation of distributed-order fractional optimal control problems," Commun. Nonlinear Sci. Numer. Simul., vol. 52, pp. 177-189, 2017. 